\title{
DIAGNÓSTICO DA ARBORIZAÇÃO DO PARQUE URBANO TUCUMÃ, EM RIO BRANCO-AC
}

\author{
AFFORESTATION DIAGNOSIS OF THE TUCUMÃ URBAN PARK, IN RIO BRANCO-AC
}

\author{
Lucélia Rodrigues Santos ${ }^{1}$, Ednéia Araújo dos Santos², Romário Mesquita Pinheiro³, \\ Evandro José Linhares Ferreira ${ }^{4}$
}

\begin{abstract}
RESUMO
O presente trabalho teve como objetivo realizar o diagnóstico da arborização da maior porção contínua do Parque Urbano Tucumã em Rio Branco-AC. Foram inventariadas todas as espécies de porte arbóreo situadas dentro da área selecionada para estudo. Verificou-se ocorrência de problemas fitossanitários (erva-de-passarinho, fungos e cupins) e danos causados pelo sistema radicular ao calçamento além de realizar a identificação botânica. Foram registrados 576 indivíduos, 69 espécies e 20 famílias botânicas. O maior número de espécies se concentrou nas famílias Fabaceae (24 spp.) e Arecaceae (14 spp.). O índice de Shannon-Wiener encontrado foi de 3,37, indicando alta diversidade de espécies. As espécies nativas corresponderam a $73,91 \%$ do total de indivíduos amostrados. Foi observado que $22,57 \%$ dos indivíduos apresentaram algum problema fitossanitário. Foram observados danos ao calçamento causado pelas raízes de $1,56 \%$ dos indivíduos registrados. Os resultados apontam a necessidade de melhor planejamento na arborização com o uso de mais famílias botânicas, melhor distribuição das espécies e medidas de controle de pragas e doenças.
\end{abstract}

Palavras-chave: Área verde urbana; Composição florística; Fitossanidade.

\section{ABSTRACT}

The present study had the aim to realize the diagnosis of the afforestation of the largest continuous portion of the Tucumã Urban Park in Rio Branco-AC. All arboreal species located within the area selected for study were inventoried. Phytosanitary issues (mistletoe, fungi and termites) and damage caused by the root system to the pavement were verified in addition to performing the botanical identification. There were registered 576 individuals, 69 species and 20 botanical families. The highest number of species was concentrated in the families Fabaceae (24 spp.) and Arecaceae (14 spp.). The Shannon-Wiener index found was 3.37, indicating high species diversity. The native species corresponded to $73.91 \%$ of the total sampled individuals. The total basal area calculated was $110.14 \mathrm{~m}^{2} /$ ha. It was noted that $22.57 \%$ of the individuals presented some phytosanitary issue. Damage to the pavement caused by the roots of $1.56 \%$ of the individuals studied was observed. The results indicate the need for better planning in the afforestation with the use of more botanical families, better distribution of the species and measures of pest and disease control.

Keywords: Urban green area; Floristic composition; Plant health.

Recebido em 06.02.2017 e aceito em 10.07.2017

1 Engenheira florestal. Mestre em Botânica. Herbário do Parque Zoobotânico da Universidade Federal do Acre- UFAC. Rio Branco/AC. Email: santos.luceliarodrigues@gmail.com

2 Engenheira florestal. Mestre em Botânica. Herbário do Parque Zoobotânico da Universidade Federal do Acre- UFAC. Rio Branco/AC. Email: edneiasantos_14@hotmail.com

3 Engenheiro agrônomo. Mestrando em Ciência e Tecnologia de Sementes. Universidade Federal de Pelotas. Pelotas/RS. Email: romario.ufacpz@hotmail.com

4 Engenheiro agrônomo. PhD. em Botânica. Pesquisador do Instituto Nacional de Pesquisas da Amazônia/INPA núcleo Acre. Rio Branco/AC. Email: evandro@inpa.gov.br 


\section{INTRODUÇÃO}

As áreas verdes urbanas possuem diversas classificações, entre elas estão a das áreas verdes com valor ecológico e uso social representadas, entre outras, pelos parques, praças e bosques urbanos (BARGOS; MATIAS, 2011). Áreas verdes podem prestar inúmeros serviços ecossistêmicos aos centros urbanos, entre eles a melhoria da qualidade do ar através do sequestro do $\mathrm{CO}_{2}$ e liberação de oxigênio, benefícios estéticos e paisagísticos criando mudanças de textura em contraposição com as áreas construídas, benefícios psicológicos relacionados com o conforto e sensação de bem estar além de servir de abrigo e fonte de alimento para a fauna (ALBERTIN et al., 2011).

Apesar de sua importância, na maior parte das cidades brasileiras a arborização é implantada sem o devido planejamento o que dificulta o seu manejo (GOMES et al., 2016). De acordo com Santos et al. (2015) o planejamento da implantação é uma das etapas mais importantes da arborização, pois a realização do plantio inadequado de árvores em áreas urbanizadas pode causar danos às redes de esgoto, elétrica e telefonia, calçamento, muros e sinalização.

O plantio inadequado durante o processo de arborização pode, entre outros transtornos, causar maior incidência de ataques por doenças e pragas. Leal, Bujokas e Biondi (2007) postulam que a infestação de árvores por erva-de-passarinho no ambiente urbano é uma das ameaças bióticas que comprometem a qualidade da arborização. Os autores afirmam ainda que por ser uma hemiparasita de crescimento vigoroso, a erva-de-passarinho pode ocupar partes da copa ou até mesmo a copa inteira de árvores e arbustos, enquanto seus haustórios (raízes modificadas) atravessam a casca do hospedeiro e absorvem sais minerais e água, prejudicando o desenvolvimento do mesmo e colocando em risco os benefícios gerados pela arborização.

Outros organismos considerados pragas são cupins e fungos. Segundo Romani et al. (2012) a adaptação dos cupins ao meio urbano favorece o processo de infestação, gerando gastos para o controle e erradicação dessa praga. Os indícios da presença desses insetos podem ser observados na forma de ninhos e túneis em partes do tronco/estipe, o que aumenta o risco de queda da árvore por diminuir a resistência mecânica da madeira (SILVA et al., 2007; HASSE et al., 2008). Os fungos por sua vez podem se tornar endêmicos infectando uma diversidade de espécies hospedeiras e podem ser encontrados associados a várias partes das plantas como folhas, raízes, botões florais, flores, caule/tronco, sementes e frutos danos expressivos às plantas (BATISTA et al., 2007; AZEVEDO et al, 2011).

SANTAMOUR-JÚNIOR (2002) cita que uma forma de reduzir os riscos da incidência de pragas e doenças em espécies vegetais no ambiente urbano é investir na diversidade de 
espécies, pois a maior heterogeneidade aumentaria a proteção. De acordo com Paiva et al. (2010) a arborização existente em rio Branco tanto nas ruas do centro quanto em outros bairros é insatisfatória tanto qualitativa quanto quantitativamente.

Tendo em vista que o diagnóstico da arborização urbana fornece informações importantes não apenas para a implantação como também para a manutenção da vegetação, o objetivo deste estudo foi realizar o diagnóstico da arborização do Parque Urbano Tucumã por meio da análise de sua diversidade e origem das espécies, presença de infestação por ervade-passarinho, cupins e fungos e danos ao calçamento causados pelo sistema radicular.

\section{MATERIAL E MÉTODOS}

O estudo foi realizado no Parque Urbano Tucumã (Coordenadas: 957'05"S; 6751'50"W; Alt.: 153m) situado na região noroeste da cidade de Rio Branco, fazendo, portanto, parte do Bioma Amazônico. A coleta de dados ocorreu entre os meses de junho a outubro de 2012. O parque foi inaugurado em dezembro de 2005 sendo bastante frequentado pela população local para a prática de esportes, alimentação e recreação contando com pistas de caminhada, ciclovias, calçadas, playgrounds, quiosques e quadras de esportes (Figura 1).
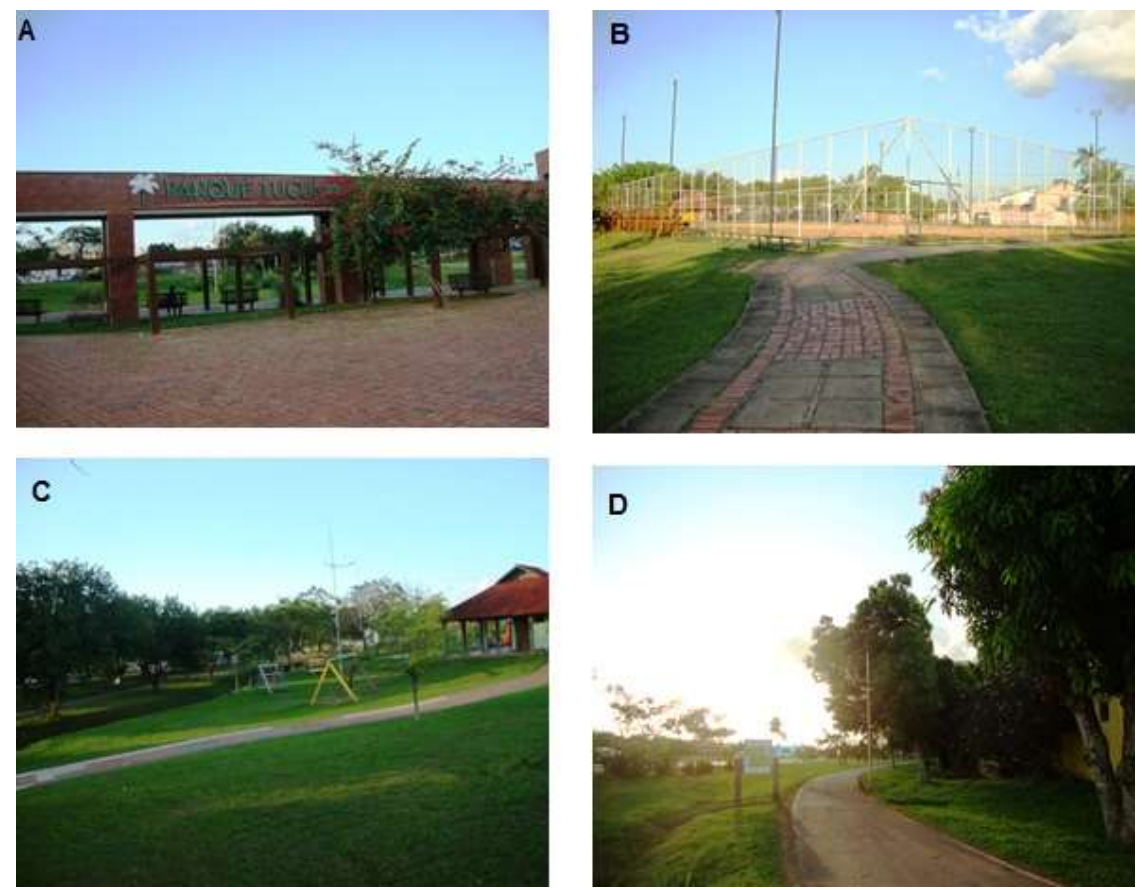

Figura 1. A. Entrada do Parque Tucumã; B. Quadra de esportes; c. Playground e quiosque; D. Pista para caminhada e ciclismo.

Figure 1. A. Tucumã Park's Entrance; B. Sports Field; C. Playground e kiosk; D. Cycling and Walking track. 
A vegetação do parque é composta por espécies plantadas durante a execução do projeto de arborização em 2005 e por algumas árvores antigas que não foram suprimidas durante o processo de avanço dos bairros de seu entorno, tendo sido incorporadas ao projeto (Figura 3).

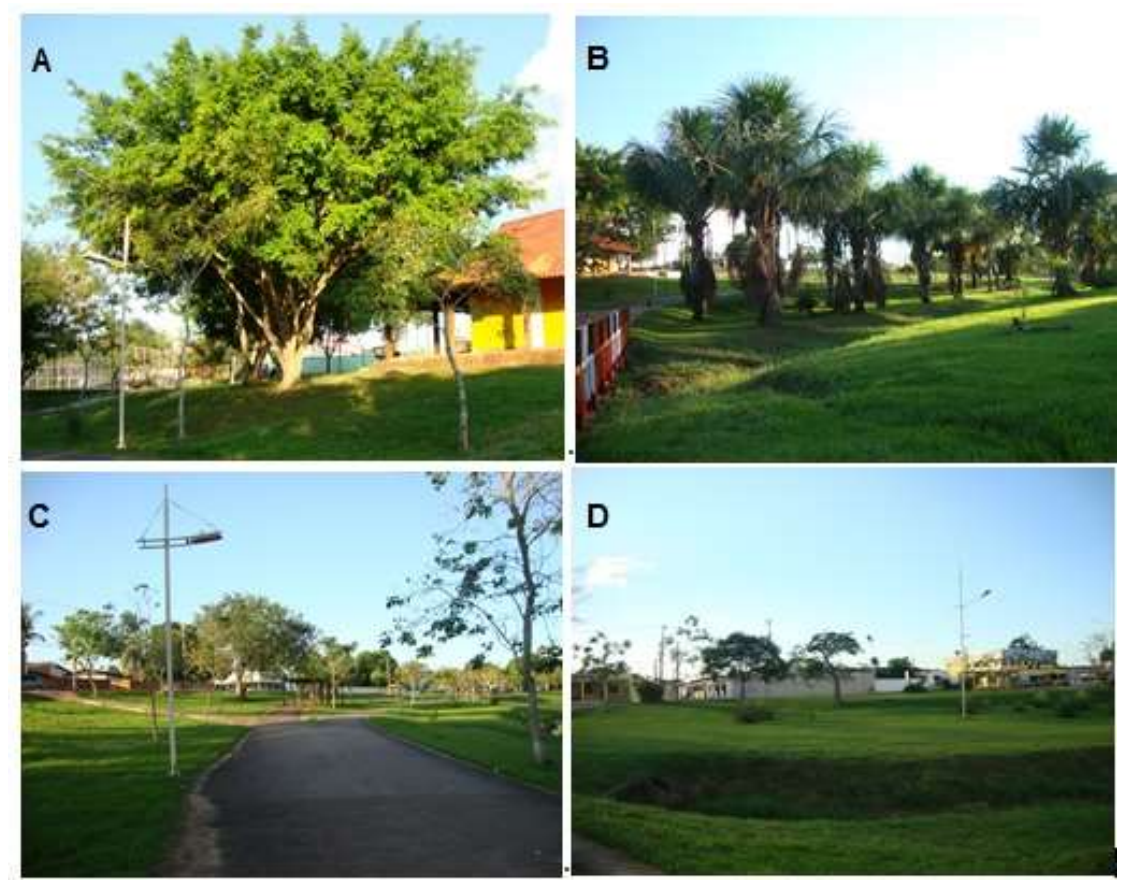

Figura 2. A. Ficus benjamina L. (ficus) plantado antes do projeto de arborização realizado em 2005 (informação fornecida por moradores); B. Indivíduos de Mauritia flexuosa L. f. (buriti) plantados em grande quantidade em 2005; C e D. Distribuição das árvores na área

Figure 2. Ficus benjamina (ficus) planted before the afforestation project made in 2005 (information provided by residents); B. Individuals from Mauritia flexuosa L. f. planted in a large quantity in 2005; C e D. Trees distribution in the area

A área ocupada pelo parque é de aproximadamente 360 hectares de extensão (OLIVEIRA; JESUS, 2011), considerando-se os canteiros ao longo da BR-364 (Figura 3). O estudo foi realizado em uma área correspondente a aproximadamente 8,7 ha $(2,4 \%$ da extensão do parque). 


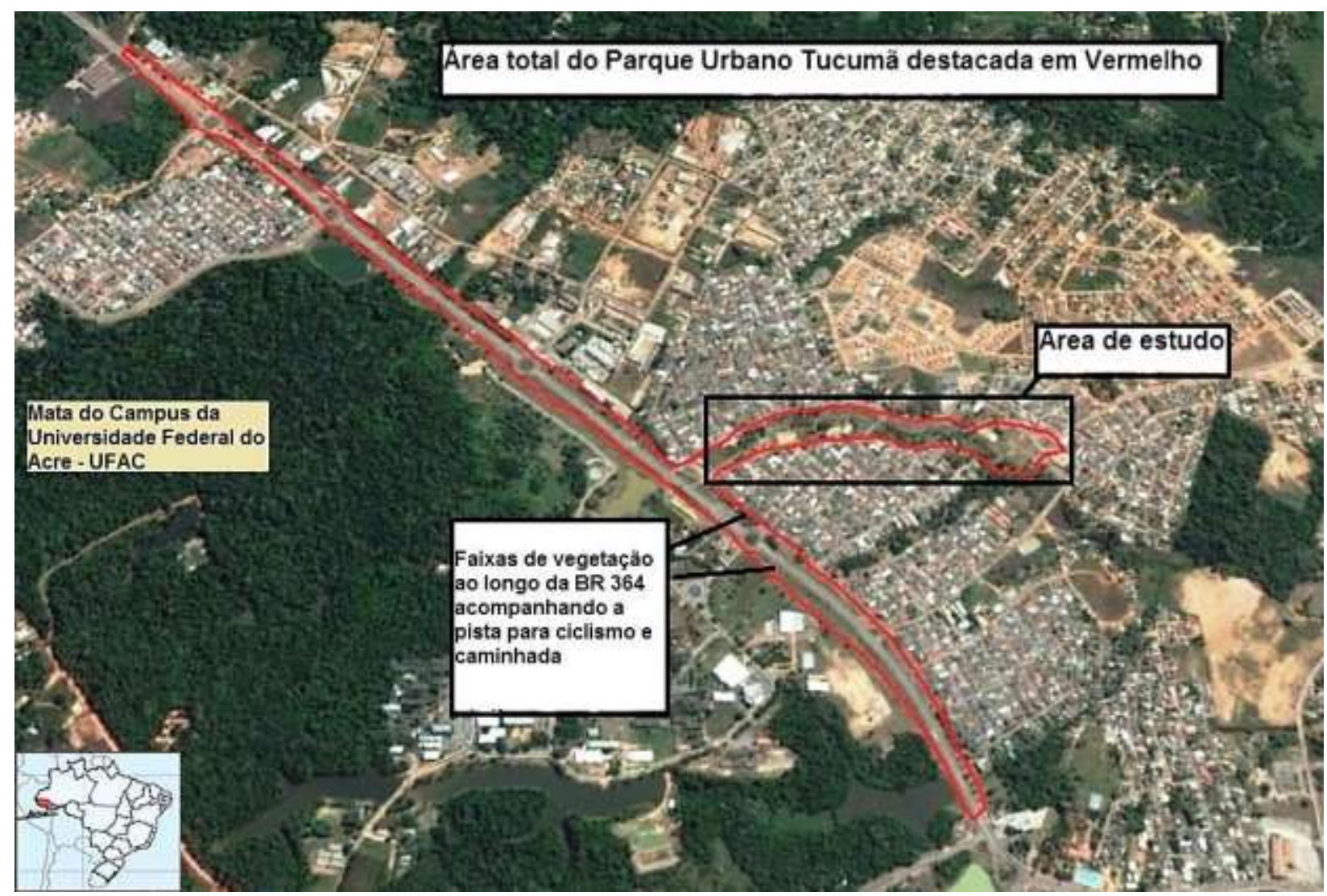

Figura 3. Área total do Parque Urbano Tucumã com destaque para a área estudo

Fonte: Google Earth $\AA^{2} 2017$

Figure 3. Total area of the Tucumã Urban Park, with emphasis on the study area

A escolha da área selecionada foi baseou-se no fato de que este local é onde foram implantados mais indivíduos arbóreos durante a realização do projeto de arborização em 2005, ao passo que nas faixas de vegetação que margeiam a rodovia o espaço foi priorizado para construção de pistas de caminhada e ciclovias onde foram implantados, comparativamente, poucos indivíduos de porte arbóreo ao longo de sua extensão. Esse método onde o levantamento não considera a área total é citado por Milano e Dalcin (2000), sendo empregado para estudos sobre arborização urbana que visem conhecer a situação da arborização e não propriamente voltados para quantificação da existência de árvores em espaços urbanos.

O inventário realizado na área foi do tipo quali-quantitativo (Paiva et al., 2010), o método utilizado foi o de caminhamento para percorrer toda a área demarcada e registrar todas as espécies de porte arbóreo ali existentes. Planilhas de campo foram utilizadas para a coleta das seguintes informações: nome popular, nome científico e estado fitossanitário. Os indivíduos foram identificados in loco por um especialista parabotânico e posteriormente, com auxílio da lista de espécies do banco de dados do Missouri Botanical Garden do The New York Botanical Garden e consulta ao acervo do Herbário do Parque Zoobotânico da Universidade Federal do Acre (UFAC/PZ), realizou-se a confirmação das identificações e da nomenclatura. 
Como parâmetro fitossociológico estudado foi escolhida a frequência de espécies (EMER;CADORIN e MELO, 2014) utilizando as fórmulas propostas por Rodrigues (1998), e para avaliar a diversidade foi empregado o índice de Shannon-Wiener (MARANHO et al, 2012). Os cálculos foram realizados no programa Microsoft Excel (2010)

(1) Frequência absoluta (FA):

$$
\frac{N_{i}}{N_{t}} \times 100
$$

(2) Frequência relativa (FR):

$$
\left(\frac{\mathbf{F A}}{\sum \mathbf{F} \mathbf{A}_{\mathbf{i}}}\right) \times 100
$$

Em que:

$\mathrm{N}_{\mathrm{i}}=$ número de indivíduos a mostrados da i-ésima espécie.

$\mathrm{N}_{\mathrm{t}}=$ número total de indivíduos.

$\sum F A_{i}=$ soma das frequências absolutas de todas as espécies amostradas.

(3) índice de Shannon-Wiener:

$$
\mathbf{H}^{\prime}=-\sum_{\mathbf{n}_{1}}^{\mathbf{S}} \frac{\mathbf{n}_{\mathbf{i}}}{\mathbf{N}} \cdot \operatorname{Ln} \cdot \frac{\mathbf{n}_{\mathbf{i}}}{\mathbf{N}}
$$

Em que:

$\mathrm{N}=$ número total de indivíduos amostrados.

$\mathrm{n}_{\mathrm{i}}=$ número de indivíduos amostrados da i-ésima espécie.

$\mathrm{Ln}=$ logaritmo neperiano.

$\mathrm{S}=$ número de espécies.

Com relação ao estado fitossanitário, verificou-se a infestação por erva-de-passarinho por meio da observação da presença dessa hemiparasita nas copas dos indivíduos (MARANHO; PAULA, 2014).

A verificação da presença de cupins no tronco/estipe também foi feita visualmente considerando a presença de seus ninhos e túneis e do pó produzido por esses insetos ao degradarem a madeira, como proposto por Silva et al. (2007).

Para identificar a infecção por fungos foi observada a presença ou ausência de sinais de colonização descritos por Ferreira (2015): apodrecimento, manchas necróticas e bolores no lenho ou nas folhas e frutos.

Os danos de raízes com o calçamento foram classificados segundo Emer, Cadorin e Melo (2014): 1- sistema radicular não aparente, 2- sistema radicular aparente, 3 - sistema radicular prejudicando a calçada, - sistema radicular prejudicando meio fio. 


\section{RESULTADOS E DISCUSSÃO}

Foram encontrados 576 indivíduos, deste total, 123 eram palmeiras. Ao todo foram contabilizados 52 gêneros e 69 espécies distribuídos em 20 famílias botânicas. Os gêneros com maior número de espécies foram: Inga com seis espécies, Acacia e Caesalpinia com três espécies cada. Fabaceae (24 spp.) e Arecaceae (14 spp.) destacaram-se como as famílias com maior número de espécies (Figura4).

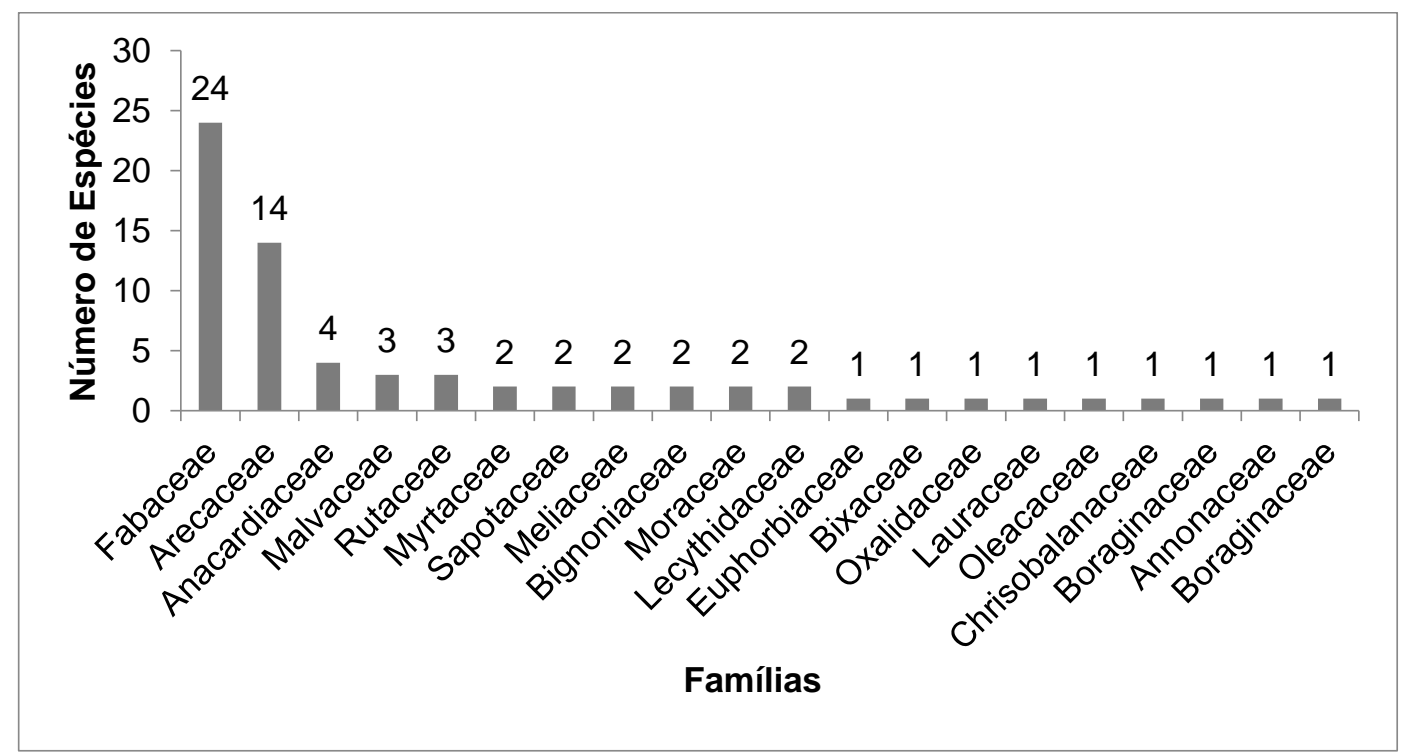

Figura 4. Número de espécies por família

Figure 4 . Species number by family

As espécies com maior número de indivíduos entre as palmeiras foram: Mauritia flexuosa L. f. (buriti) (61), Acrocomia aculeata (Jacq.) Lodd. ex Mart (macaúba) (22), Attalea phalerata Mart. ex Spreng (uricuri) (10) e Cocos nucifera L. (coco) (8). Entre as arbóreas, as espécies que apresentaram o maior número de indivíduos foram: Licania tomentosa (Benth.) Fritsch (oití) (55), Handroanthus serratifolius (Vahl) S.O.Grose (ipê-amarelo) (52), Caesalpinia pluviosa var. peltophoroides (Benth.) G.P.Lewis (sibipiruna) (48) e Mangifera indica L. (mangueira) (32). Destas espécies mais abundantes apenas $M$. indica é exótica, 73,91\% das espécies encontradas no parque são nativas do Brasil (Tabela 1). Segundo Paiva et al. (2010) o uso de espécies nativas na arborização é altamente recomendado por sua melhor adaptação, além de garantir a manutenção de relações ecológicas de dispersão de sementes e pólen envolvendo a fauna local. 
Tabela 1. Ordenação das espécies encontradas no Parque Tucumã, Rio Branco, AC, em ordem decrescente de número de indivíduos

Table 1. Ordering of the species found in the Tucumã Park, Rio Branco, Acre, in decreasing order of number of individuals

\begin{tabular}{|c|c|c|c|c|c|}
\hline Espécie & Nome popular & Família & $\mathbf{N}$ & $\begin{array}{l}\mathrm{Fr} \\
(\%)\end{array}$ & 0 \\
\hline Mauritia flexuosa L. f. & Buriti & Arecaceae & 61 & 10,59 & NB \\
\hline Licania tomentosa (Benth.) Fritsch & Oití & Chrysobalanaceae & 55 & 9,55 & NB \\
\hline Handroanthus serratifolius (Vahl) S.O.Grose & Ipê-amarelo & Bignoniaceae & 52 & 9,03 & NB \\
\hline $\begin{array}{l}\text { Caesalpinia pluviosa var. peltophoroides (Benth.) } \\
\text { G.P.Lewis }\end{array}$ & Sibipiruna & Fabaceae & 48 & 8,33 & NB \\
\hline Mangifera indica L. & Mangueira & Anacardiaceae & 32 & 5,56 & $\mathrm{E}$ \\
\hline Clitorea racemosa Benth.) & Paliteiro & Fabaceae & 30 & 5,21 & NB \\
\hline Bauhinia forficata Link & Pata-de-vaca & Fabaceae & 28 & 4,86 & NB \\
\hline Handroanthus heptaphyllus (Vell.) Mattos & Ipê-roxo & Bignoniaceae & 27 & 4,69 & NB \\
\hline Acrocomia aculeata (Jacq.) Lodd. ex Mart. & Macaúba & Arecaceae & 22 & 3,82 & NB \\
\hline Acacia farnesiana (L.)Willd. & Acácia-amarela & Fabaceae & 17 & 2,95 & $\mathrm{E}$ \\
\hline Achras sapota L. & Sapotí & Sapotaceae & 16 & 2,78 & $E$ \\
\hline Ficus benjamina L. & Benjamim & Moraceae & 15 & 2,60 & $\mathrm{E}$ \\
\hline Inga fagifolia (L.) Willd. ex Benth. & Ingá-mirim & Fabaceae & 15 & 2,60 & NB \\
\hline Anacardium occidentale L. & Cajueiro & Anacardiaceae & 14 & 2,43 & NB \\
\hline Attalea phalerata Mart. ex Spreng. & Uricuri & Arecaceae & 10 & 1,74 & NB \\
\hline Psidium guajava L. & Goiabeira & Myrtaceae & 9 & 1,56 & NB \\
\hline Cocos nucifera L. & Coqueiro-da-praia & Arecaceae & 8 & 1,39 & NB \\
\hline Acacia sp. & Brinco-de-ouro & Fabaceae & 7 & 1,22 & $\mathrm{E}$ \\
\hline Inga sessilis (Vell.) Mart. & Ingá-de-macaco & Fabaceae & 7 & 1,22 & NB \\
\hline Theobroma grandiflorum (Willd. ex Spreng.) Schum & Cupuaçuzeiro & Malvaceae & 6 & 1,04 & NB \\
\hline Delonix regia (Bojer ex Hook.) Raf. & Flamboiã & Fabaceae & 6 & 1,04 & $E$ \\
\hline Pithecellobium sp. & Jurema-da-folha-miúda & Fabaceae & 5 & 0,87 & NB \\
\hline Persea americana Mill. & Abacateiro & Lauraceae & 4 & 0,69 & $\mathrm{E}$ \\
\hline Dypsis lutescens (H.Wendl.) Beentje \& J.Dransf & Areca-bambu & Arecaceae & 4 & 0,69 & $E$ \\
\hline Calliandra sp. & Bordão-de-velho & Fabaceae & 4 & 0,69 & NB \\
\hline Annona squamosa L. & Fruta-do-conde & Annonaceae & 4 & 0,69 & $\mathrm{E}$ \\
\hline Syagrus oleraceae (Mart.) Becc. & Guariroba & Arecaceae & 4 & 0,69 & NB \\
\hline Attalea butyracea (Mutis ex L.f.) Wess.Boer & Jaci & Arecaceae & 4 & 0,69 & NB \\
\hline Spondias mombin L. & Cajá & Anacardiacea & 3 & 0,52 & NB \\
\hline Copaiba langsdorfii (Desf.) Kuntze & Copaíba & Fabaceae & 3 & 0,52 & NB \\
\hline Inga sp.2 & Ingá-olho-de-boi & Fabaceae & 3 & 0,52 & NB \\
\hline Swietenia macrophylla King & Mogno & Meliaceae & 3 & 0,52 & NB \\
\hline Caryota urens L. & Palmeira-rabo-de-peixe & Arecaceae & 3 & 0,52 & $E$ \\
\hline Bactris gasipaes Kunth. & Pupunha-comum & Arecaceae & 3 & 0,52 & NB \\
\hline Ceiba pentandra (L.) Gaertn. & Samaúma & Malvaceae & 3 & 0,52 & NB \\
\hline Citrus reticulata Blanco & Tangerina & Rutaceae & 3 & 0,52 & $E$ \\
\hline Syzygium cumini (L.) Skeels. & Azeitona & Myrtaceae & 2 & 0,35 & $E$ \\
\hline Parkia sp. & Faveira & Fabaceae & 2 & 0,35 & NB \\
\hline Cordia alliodora (R. F.) Chaw. & Freijó & Boraginaceae & 2 & 0,35 & NB \\
\hline Inga velutina Willd. & Ingá-peluda & Fabaceae & 2 & 0,35 & NB \\
\hline Caesalpinia ferrea Mart. & Jucá & Fabaceae & 2 & 0,35 & NB \\
\hline Pouteria caimito (Ruiz \& Pav.) Radlk.Roem\&Schult & Abiu-amarelo & Sapotaceae & 1 & 0,17 & NB \\
\hline
\end{tabular}


Acacia podalyriifolia A.Cunn. ex G.Don

Euterpe oleraceae Mart.

Rollinia exsucca (Dun.) DC.

Theobroma cacao L.

Schizolobium amazonicum Hub.

Averrhoa carambola L.

Bertholletia excelsa H.B.K

Cedrela odorata $L$.

Rollinia mucosa (Jacq.) Baill

Amburana cearensis (Fr. All.) A.C. Smith

Dimorphandra mollis Benth

Senna silvestris (Vell.) H.S.Irwin \& Barneby

Annona muricata L.

Inga sp. 1

Inga edulis Mart.

Artocarpus heterophyllus Lam.

Phytelephas macrocarpa Ruiz \& Pav.

Pithecellobium Diversifolium Benth

Citrus aurantifolia (Christm.) Swingle

Cochlospermum orinocense (Kunth) Steud.

Livistona chinensis (Jacq.) R.Br. ex Mart.

Caesalpinia leiostachya (Benth.) Ducke

Hevea brasiliensis (Willd. ex A. Juss.) Müll. Arg.

Vatairea sericea (Ducke) Ducke

Spondias sp.

Couratari macrosperma A.C.Sm.

Astrocaryum aculeatum G.Mey.

\begin{tabular}{|c|c|c|c|c|}
\hline Acácia & Fabaceae & 1 & 0,17 & $E$ \\
\hline Açaí-de-touceira & Arecaceae & 1 & 0,17 & NB \\
\hline Ata-brava & Annonaceae & 1 & 0,17 & $\mathrm{NE}$ \\
\hline Cacaueiro & Malvaceae & 1 & 0,17 & $\mathrm{~N}$ \\
\hline Canafístula & Fabaceae & 1 & 0,17 & $\mathrm{~N}$ \\
\hline Carambola & Oxalidaceae & 1 & 0,17 & $E$ \\
\hline Castanheira & Lecythidaceae & 1 & 0,17 & $\mathrm{NE}$ \\
\hline Cedro-rosa & Meliaceae & 1 & 0,17 & 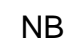 \\
\hline Condessa & Annonaceae & 1 & 0,17 & $\mathrm{NE}$ \\
\hline Cumarú-de-cheiro & Fabaceae & 1 & 0,17 & $\mathrm{~N}$ \\
\hline Faveira-da-folha-miúda & Fabaceae & 1 & 0,17 & $\mathrm{~N}$ \\
\hline Faveira-São-João & Fabaceae & 1 & 0,17 & $\mathrm{~N}$ \\
\hline Graviola & Annonaceae & 1 & 0,17 & $E$ \\
\hline Ingá & Fabaceae & 1 & 0,17 & $\mathrm{~N}$ \\
\hline Ingá-de-corda & Fabaceae & 1 & 0,17 & $\mathrm{~N}$ \\
\hline Jaqueira & Moraceae & 1 & 0,17 & \\
\hline Jarina & Arecaceae & 1 & 0,17 & $\mathrm{~N}$ \\
\hline Jurema & Fabaceae & 1 & 0,17 & $\mathrm{~N}$ \\
\hline Limão-galego & Rutaceae & 1 & 0,17 & E \\
\hline Pacotê & Bixaceae & 1 & 0,17 & $\mathrm{E}$ \\
\hline Palmeira-de-leque & Arecaceae & 1 & 0,17 & $\mathrm{E}$ \\
\hline Pau-ferro & Fabaceae & 1 & 0,17 & $\mathrm{~N}$ \\
\hline Seringueira & Euphorbiaceae & 1 & 0,17 & $\mathrm{~N}$ \\
\hline Sucupira & Fabaceae & 1 & 0,17 & $N$ \\
\hline Taperebá & Anacardiacea & 1 & 0,17 & $\mathrm{~N}$ \\
\hline Tauarí & Lecythidaceae & 1 & 0,17 & $\mathrm{~N}$ \\
\hline \multirow[t]{2}{*}{ Tucumã } & Arecaceae & 1 & 0,17 & \\
\hline & & 576 & 100 & \\
\hline
\end{tabular}

Legenda: $\mathrm{N}=$ número de indivíduos, $\mathrm{Fr}=$ frequência, $\mathrm{NB}=$ nativa do Brasil, $\mathrm{E}=$ exótica, $\mathrm{O}=$ Origem Key: $\mathrm{N}=$ individuals number, $\mathrm{Fr}=$ relative frequency, $\mathrm{NB}=\mathrm{Brazil}$ native, $\mathrm{E}=$ exotic, $\mathrm{O}=$ origin

O índice de diversidade de Shannon-Wiener encontrado foi 3,37 valor próximo ao máximo para esse parâmetro que é de 3,5 indicando uma elevada diversidade de espécies para a área analisada. Esse resultado foi próximo ao encontrado por Maranho e Paula (2014) no Campus da Universidade Federal do Acre, também situado no município de Rio Branco $\left(H^{\prime}=3,95\right)$, segundo os autores $77 \%$ das 128 espécies encontradas no local são nativas do Brasil, percentual este que também se aproximou do encontrado neste estudo (73,91\%).

As famílias Fabaceae e Arecaceae foram responsáveis por $34,78 \%$ e 20,28\% das espécies registradas, estas famílias são citadas em outros estudos sobre arborização urbana e aparecem como predominantes nos levantamentos realizados por Freitas, Pinheiro e Abrahão (2015) em praças do bairro da Tijuca-RJ (59,3\% e 8\% respectivamente) e Gomes et al. (2016) no município de Macapá-AM (29,16\% e 12,5,\%, respectivamente). Gomes et al. (2016) afirmam que é frequente a predominância de espécies da família Fabaceae em áreas verdes urbanas, o que demonstra a importância desta família na arborização. No entanto, deseja-se que exista 
alta diversidade no espaço urbano, segundo Santamour-Júnior (2002) o recomendável é que não se exceda $10 \%$ da mesma espécie, $20 \%$ do mesmo gênero e $30 \%$ da mesma família, o resultado encontrado aponta um excesso de espécies da família Fabaceae. De acordo com Araújo et al. (2012) as árvores que ocupam o espaço urbano estão mais sujeitas ao ataque de pragas e doenças em decorrência do ambiente antropizado e uma maior diversidade pode diminuir os riscos à fitossanidade local. Santos Júnior e Costa (2014) postulam que há espécies que ficam mais visadas no paisagismo em determinadas épocas, fazendo com que ocorra plantio massivo de poucas espécies em detrimento de outras, o que explica a grande quantidade de determinadas espécies em áreas urbanas.

Os resultados do inventário qualitativo indicaram que dos 576 indivíduos registrados $130(22,3 \%)$ apresentaram algum problema fitossanitário (Figura 3). As espécies com maior número de indivíduos infestados por erva-de-passarinho foram Bauhinia forficata (75\%), Caesalpinia pluviosa (43,75\%) e Achras sapota L. (37,5\%).

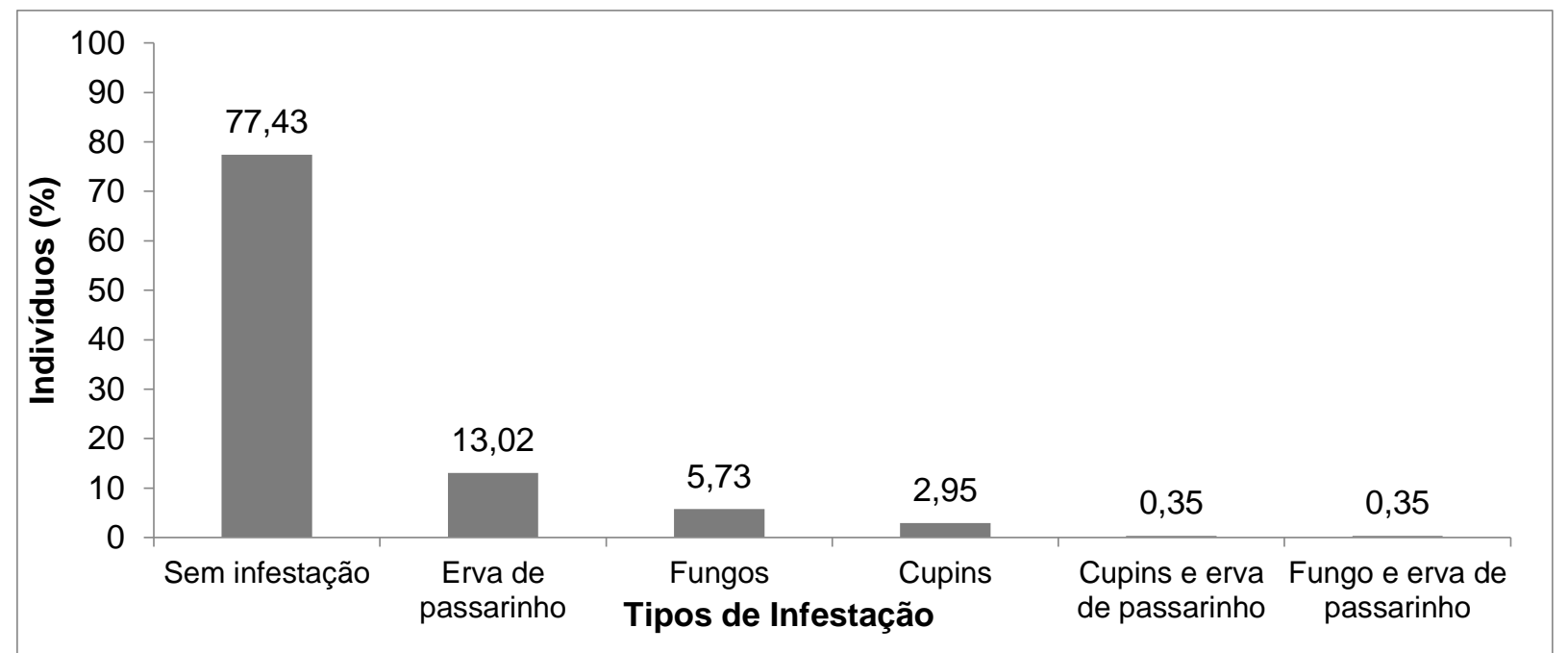

Figura 5. Problemas fitossanitários encontrados nos indivíduos amostrados em um fragmento do Parque Urbano Tucumã, Rio Branco, Acre

Figure 5. Phytosanitary problems found in individuals sampled in a fragment of the Tucumã Urban Park, Rio Branco, Acre

O resultado para a infestação por erva-de-passarinho ficou abaixo do encontrado em outros estudos realizados neste mesmo município, Maranho e Paula (2014) encontraram uma porcentagem de infestação por erva-de-passarinho de $17 \%$ no campus da Universidade Federal do Acre, em Rio Branco. Rosa e Paiva (2009) em um estudo avaliando o nível de infestação por erva-de-passarinho em árvores do centro da cidade de Rio Branco encontraram uma porcentagem de $27 \%$ de indivíduos parasitados, sendo que Caesalpinia pluviosa também estava entre as espécies mais atacadas, esta espécie é bastante utilizada na arborização da 
cidade e sua característica de apresentar uma copa frondosa e sempre-verde pode facilitar a permanência de hemiparasitas, sendo recomendada manutenção constante.

A espécie mais afetada por cupins foi Ficus benjamina (2,6\%). Não foi encontrada na literatura nenhuma indicação de que esta espécie seja mais susceptível ao ataque por cupins do que outras, no entanto, Albertin et al. (2011) afirmam que o fator idade pode estar relacionado a uma maior incidência de ataque por esses insetos. No presente estudo observou-se que a maioria dos indivíduos de Ficus benjamina eram árvores que já se encontravam no local antes do plantio de mudas de 2005, sendo, portanto, árvores de mais idade o que pode ter favorecido a infestação.

As três espécies mais infestadas por fungos foram Licania tomentosa, Handroanthus serratifolius e Mauritia flexuosa (9,54\%, 9,04\% e 8,19\%, respectivamente), as três são as de maior ocorrência no parque (61, 55 e 52 indivíduos respectivamente) o que pode explicar o grande número de indivíduos infectados comparados aos das demais espécies. Ferreira (2015) cita como sinais de doenças causadas por fungos as manchas foliares, as podridões, o cancro (no tronco) e a murcha vascular. De acordo com a mesma autora, a infecção por fungos pode causar desde o atraso no desenvolvimento dos indivíduos jovens até a morte de árvores adultas, destacando a importância do diagnóstico para a adoção de medidas que evitem prejuízos à arborização urbana em decorrência desses organismos.

$\mathrm{Na}$ maioria dos indivíduos (85,76\%) o sistema radicular não apresentava conflito com o calçamento (Figura 6).

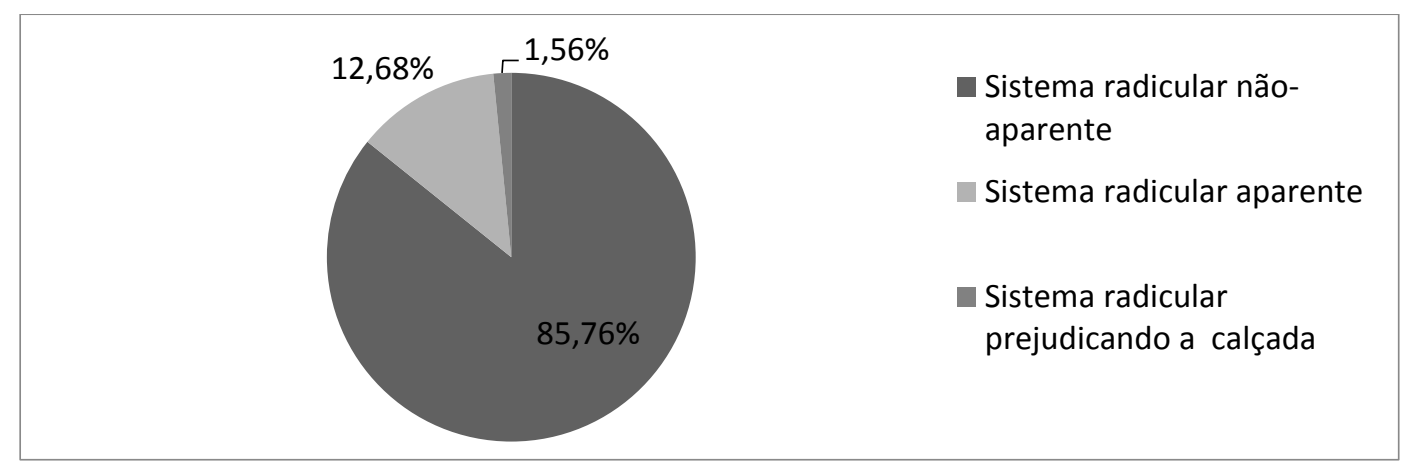

Figura 6.Situação do sistema radicular das espécies avaliadas no Parque Urbano Tucumã, Rio Branco-AC Figure 6. Situation of the root system of the evaluated species in the Tucumã Urban Park, Rio Branco-AC

Emer, Cadorin e Melo (2014) verificaram que na arborização do bairro Jardim Primavera em Pato Branco - PR 88\% das árvores não exibiam raízes aparentes, 7\% possuíam raízes prejudicando a calçada e 4\% tinham raízes aparente. Segundo esses autores, raízes expostas podem se tornar um transtorno mesmo que não afetem áreas calçadas, pois podem se constituir em obstáculos para pedestres e aumentar a dificuldade de locomoção para pessoas com mobilidade reduzida. 
A ausência de danos causados pelas raízes aparentes ocorreu devido ao fato de que estas estavam localizadas fora das áreas longe da estrutura urbana. As espécies que apresentaram raízes causando danos ao calçamento foram Mangifera indica (22,22\%), Clitoria racemosa (22,22\%), Acacia farnesiana (33,33\%) e Parkia sp. (22,22\%). Entre as principais espécies que apresentaram raízes expostas sem, no entanto, prejudicar estruturas estão Clitoria racemosa (30,14\%), Ficus benjamina, (16,44\%), Mangifera indica (8,22\%) e Acrocomia aculeata (5,48\%). F. benjamina e $M$. indica são citadas nos estudos de Santos et al. (2015) e Maranho e Paula (2014), respectivamente, como causadoras de danos ao calçamento devido seu sistema radicular agressivo. Ainda de acordo com Santos et al. (2015) nas cidades brasileiras é comum o plantio de espécies que possuem sistema radicular superficial e com pouco desenvolvimento radicular em profundidade, o que provoca problemas em calçadas e canteiros podendo inclusive comprometer a estrutura de imóveis próximos.

\section{CONCLUSÕES}

A análise da arborização de uma área do Parque Urbano Tucumã indicou um índice de diversidade de espécies alto, sendo a maioria das espécies nativas. No entanto, o número de famílias botânicas poderia ser maior para atender aos critérios recomendados para reduzir os riscos de ataque de pragas e doenças aos indivíduos, a distribuição das espécies também poderia ser mais adequada dando mais harmonia ao parque, uma vez que muitas espécies apresentam menos de $1 \%$ de frequência ao passo que outras foram plantadas em grandes quantidades, como foi o caso de Mauritia flexuosa. A porcentagem de espécies nativas é superior a de exóticas, no entanto, levando em consideração a riqueza especifica encontrada na região, as espécies nativas poderiam figurar em maior quantidade do que a encontrada.

A infestação da vegetação por erva-de-passarinho, fungos e cupins apontam a necessidade de cuidados para erradicação dessas pragas. O conflito do sistema radicular com áreas concretadas indica a importância da adequação da área construída com manutenção do calçamento aumentando o espaço físico para as raízes.

Recomenda-se a criação de um plano de monitoramento para a arborização do Parque Urbano Tucumã, com inspeção sistemática dos indivíduos fazendo o controle fitossanitário e evitando possíveis danos às áreas construídas. 


\section{AGRADECIMENTOS}

Ao Sr. João Bosco Queiroz, especialista parabotânico, pelo auxílio nas identificações em campo.

\section{REFERÊNCIAS}

ALBERTIN R. M, DE ANGELIS F, DE ANGELIS NETO R, DE ANGELIS B. L. D. Diagnóstico quali-quantitativo da arborização viária de Nova Esperança, Paraná, Brasil. Revista da Sociedade Brasileira de Arborização Urbana, Piracicaba, v. 6, n. 3, p. 128-148, 2011.

ARAÚJO, A. C. B.; GRACIOLI, C. R.; GRIMM, E. L.; LONGHI, S. J. Avaliação da florística, do porte e da fitossanidade atual da arborização do Parque Internacional de Sant'ana do Livramento/Rivera, Brasil/Uruguay. Revista da Sociedade Brasileira de Arborização Urbana, Piracicaba, v. 7, n. 1, p. 112-125, 2012.

AZEVEDO, G. B.; FERREIRA, G. F. P.; SOUSA, G. T. O.; NOVAES, Q. S. Fungos associados a árvores e arbustos em vias públicas de Vitória da Conquista, Bahia. Enciclopédia Biosfera, Goiânia, v. 7, n. 12, p. 2-14, 2011.

BARGOS, D. C.; MATIAS, L. F. Áreas verdes urbanas: Um estudo de revisão e propostas conceituais. Revista da Sociedade Brasileira de Arborização Urbana, Piracicaba, v. 6, n. 3, p.172-188, 2011.

BATISTA, T. F. C.; ALVES. K. F.; SANTOS FILHO, B. G.; RODRIGUES, R. C.; OLIVEIRA, F. C.; TAVARES, A. E. B.. Ocorrência de fungos e nematoides fitopatogênicos em áreas reflorestadas pela Petrobrás oriundas da exploração petrolífera no município de Coari (AM). Revista de Ciências Agrárias, Recife, n.47, p.163-171, 2007.

EMER,A.; CADORIN, D. A.; MELO, N. A. Avaliação quali-quantitativa da arborização do bairro Jardim Primavera, na cidade de Pato Branco-PR. Revista Científica Anap Brasil, Tupã, n. 9, v. 7, 19-32, 2014.

FERREIRA, M. A. Doenças bióticas e abióticas na arborização urbana. In: Circuito Cemig de Arborização Urbana, 5, Caxambu, 2015. Mini curso. Caxambu: Universidade Federal de Lavras, 2015.

FREITAS, W. K.; PINHEIRO, M. A. S.; ABRAHÃO, L. L. F. Análise da arborização de quatro praças no Bairro da Tijuca, RJ, Brasil. Floresta e Ambiente, Seropédica, v. 22, n. 1, p. 23-31, 2015.

GOMES, E. M. C.; RODRIGUES, D. M. S.; SANTOS, J. T.; BARBORSA, E. J. Análise qualiquantitativa de uma praça urbana no norte do Brasil. Nativa, Sinop, v. 4, n. 3, p. 179-186, 2016.

HASSE, I.; SHINOSAKA, T. J.; SILVA, L. M. Avaliação da presença de cupins na arborização da região central de Pato Branco, PR. Revista da Sociedade Brasileira de Arborização Urbana, Piracicaba, v. 3, n. 1, p. 9-18, 2008.

LEAL, L. BUJOKAS, W. M.; BIONDI, D. Análise da infestação de erva-de-passarinho na arborização das ruas de Curitiba, PR. Floresta, Curitiba, v. 36, n. 3, 2006. 
MARANHO, A. S.; PAULA, S. R. P.; LIMA, E.; PAIVA, A. V.; ALVES, A. P.; NASCIMENTO, D. O. N. Levantamento censitário da arborização urbana viária de Senador Guiomard, Acre. Revista da Sociedade Brasileira de Arborização Urbana, Piracicaba, v. 7, n. 3, p. 44-56, 2012.

MARANHO, A. S.; PAULA, S. R. P. Diversidade em uma área verde urbana: avaliação qualitativa da arborização do campus da Universidade Federal do Acre, UFAC, Brasil. Revista Agroambiente, Boa Vista, v. 8, n. 3, p. 404-415, 2014.

MILANO M. S.; DALCIN, E. Arborização de vias públicas. Rio de Janeiro: Light, 2000, 226 p.

OLIVEIRA, K. A.; JESUS, I. S. Espacialização e quantificação das áreas verdes no perímetro urbano no município de Rio Branco, Acre. In: SIMPOSIO BRASILEIRO DE SENSORIAMENTO REMOTO, 15, 2011, Anais... Curitiba: INPE. 2011. p. 0877.

PAIVA, A. V.; LIMA, A. B. M.; CARVALHO, A.; JUNIOR, A. M.; GOMES, A.; MELO, C. S.; FARIAS, C. O.; REIS, C.; BEZERRA, C.; JUNIOR, E. A. S.; MACEDO, E.; LIMA, E. S.; SOBRINHO, F.; SILVA, F. M.; BONFIM, J. C.; JUNIOR, L. S.; CORREA, M.; DUMONT, M. L.; ISAAC JUNIOR, M. A.; PANTOJA, N. V.; DAVILA, R. M.; GABRIEL, R.; SILVA, R. A.; CUNHA, R. M. OLIVEIRA, R. S.; DIAS, R.; NICHELI, S. P.; COSTA, S.; SOUZA, T. C.; PEREIRA, T. F.; CASTELO. Z; FERRARI, Z. S. Inventário e diagnóstico da arborização urbana viária de Rio Branco, AC. Revista da Sociedade Brasileira de Arborização Urbana, Piracicaba, v. 5, n. 1, p. 144-159, 2010.

RODRIGUES, R. R. Métodos fitossociológicos mais usados. São Paulo: Casa da Agricultura, 1988. Separata.

ROMANI, G. N.; GIMENES, R.; SILVA, M. T.; PIVETA, K. F. L.; BATISTA, G. S. Análise qualiquantitativa da arborização da Praça XV de Novembro em Ribeirão Preto-SP, Brasil. Revista Arvore, Viçosa, v. 36, n. 3, 479-487, 2012.

ROSA, S. K.; PAIVA, A. V. Níveis de infestação de ervas-de-passarinho em árvores nas áreas públicas do Centro de Rio Branco. In: Congresso Brasileiro de Arborização Urbana, 13, 2009, Rio Branco. Resumos. Rio Branco: SBAU, 2009.

SANTAMOUR JÚNIOR, F. S. Trees for urban planting: diversity uniformity, and common sense. In: Elevitch, C. R. (Org.).The overstory book: cultivating connections with trees. Holualoa: Permanent: Agriculture Resources, 2002. p. 396-399.

SANTOS, C. Z. A.; FERREIRA, R. A.; SANTOS, L. R.; SANTOS, L. I.; GOMES, S. H.; GRAÇA, D. A. S. Análise qualitativa da arborização urbana de 25 vias públicas da cidade de Aracaju. Ciência Florestal, Santa Maria, v. 25, n. 3, p. 751-763, 2015.

SANTOS JÚNIOR, A.; COSTA, L. M. Espécies empregadas na arborização urbana do Bairro Santiago, Ji-Paraná/RO. Revista da Sociedade Brasileira de Arborização Urbana, Piracicaba, v. 9, n. 1, p. 78-91, 2014.

SILVA, L. M.; MOCCELLIN, R.; WEISHEIMER, D. I. ; ZBORALSKI, A. R.; FONSECA, L.; RODIGHIERO, D. A. Inventário e sugestões para arborização em via pública de Pato Branco/PR. Revista da Sociedade Brasileira de Arborização Úrbana, Piracicaba, v. 2, n. 1 p. 101-108, 2007. 